\title{
RECENT TECHNICAL OF ADDED SAMPLE ADAPTIVE OFFSET FOR HEVC
}

\author{
Yasir Abdulraheem Ahmed, Ali Jasim Ghaffoori, \\ Department of Electrical Power Techniques Engineering, Al-Ma'moun University \\ alobaidi.yasir99@gmail.com, ali_jk1972@yahoo.com
}

\begin{abstract}
Video compression uses encoding to convert video files into smaller files for efficient storage and transmission in image processing. A decoder reconstructs the received data into a representation of the original video. This research presents recently added in-loop as a filtering technique for extension of sample adaptive offset (SAO) in High-Efficiency Video Coding (HEVC). The principle of SAO is to lessen pixel errors caused by the compression process. Through this an average data reduction up to $1.43 \%$ in Low delay configuration and $1.03 \%$ in Random access configuration.
\end{abstract}

Keywords: HEVC, SAO

Paper History :(Received :27-12-2016; Accepted :23-52019)

\section{Introduction}

The work together of joint project latest video codec of the ITU-T Video Coding Experts Group (VCEG) and standardization organizations the ISO/IEC Moving Picture Experts Group (MPEG) in the context referred to as "joint cooperative partnership group to Video Coding (JCT-VC)may be presents the (HEVC) standard. The main objective of the (HEVC) [2] reducesthe bit rate of $50 \%$ compared with H.264/AVC [3], under identical video quality. In (HEVC), then still takes place based on the block as inter/intra prediction, quantization, and transmutation. In the former video coding standards applied a deblocking filter (DBF) reconstructed block border to minimize blocking monuments.In addendum to DBF, use new technology referred to as SAO filter after DBF to adapt the rebuild samples.SAO is helpful of transmutation coefficients which principally come from the quantization errors to reduce the ringing monuments. The paper is organized as follows in section 2 describe the (HEVC), in section 3 explain (SAO), in section 4 show experiments and results, in section 5 concludes this research.

\section{High-Efficiency Video Coding}

The (HEVC) is the latest videotechnology for compression is designed including coding efficiency, easy transportation system integration, data loss resilience, and parallel processing architectures implement ability exploitation[2]. The video coding of (HEVC) by followed 2-D converting coding is used the traditional block-based hybrid approach, i.e., inter-/intra-picture prediction.
Figure (1) shows the HEVC encoder block diagram [4]. The HEVCencoding algorithmproduced bitstream when partitioned into block-shaped regionsfor the picture to be encoded. The first frame with the help of intrapicture prediction alone of the encoded video sequence. The pictures coded using inter-picture prediction mode exploiting the temporal redundancies between the random access points or the remaining sequence pictures. Interpicture prediction comprises which consists of the motion vector (MV) and the specific reference picture of choosing motion data, for use to predict each block samples. Mode decision data and motion vectors as side data are transmitted to the decoder and using this information both encoder and decoder produce identical inter-picture prediction signals. Theresidual signal is generated for both intra and inter-prediction blocks with the difference between the original block and its predicted block. The linear spatial transform to transformed residual signal, the resulting with the prediction information along is scaled, quantized, entropy coded and transmitted.

\section{Sample Adaptive Offset}

The (SAO) rely on the kind of the sample may using various offsets sample by sample in a zone, and are adapted parameters from zone to zone [5]. The zone size is fixed to one coding tree block to achieve low torpor encoder and reduce the requirements barrier; then diverse coding tree units can be merged combined for share sample adaptive offset parameters to minimize side information [6]. The two kinds of SAO which could meet the requirements the edge of the offset (EO) and the band offset (BO)of low complexity in the HEVC. For (EO) the sample arrangement is depending on samples as current and neighboring but in the (BO), depending on the values of the sampleas a comparison between them. Note that each component of the color may have its own SAO parameters [7].

In the band offset mode, the adaptive offset ofsample stratifies formality to the values of the sample that only on a sample density basis and not on the sample district. It is divided into three ' band'sdensity': (a) low, transition and high. The field of the entire value 32 binsquantity of every ingredient, explained by the five most significant bits from sample value. The bin lowest index $i_{0}$ as a position of the SAO band in the bitstream of the transition b the and is signaled. The transition band are indicated for individual sample intensity offset value in the bitstream as wtheidth of four bins. These values adaptation dedicated to density values in the bandcan be pa ositive or negative offset for each component and not changein ranges high or low density samples with values. 
An example of positioning the band (SAO) is given in Figure (2) [8].

Figure (3) shows four the 1-D three-pixel patterns, then to classify pixels based on their edge direction the (EO) uses one of the four 1-D patterns [9]. Theapply to different regions encoder selects (BO) or (EO) of the picture, and it can also signal that both selects don't use in a particular region of the picture.

\section{Experiments and Results}

The proposed method can improve the filter in HEVC shown in figure (4). Spacious experimentsand gauge the performance are executed. Evolutions are performed in reference 2D-(HEVC) codec version (HM-KTA)[10]. All tests are executed which describe configuration default encoder through the International Organization of Standardization in keeping with (JCT-VC) Common check Conditions for assessment of 2D codec performance applied.The coding efficiency at four points of operative $(\mathrm{QP}=37 ; 32 ; 27 ; 22)$ is measured, the average rate reduction computing in compared to the (HEVC) while not (SAO)[11] and in Table 1 the results will be summarized. Figure (5) show bitrate reduction of the BQSequare and PartyScene sequences in random access configuration. The for all check sequences for (HEVC) with (SAO) and without, YPSNR values and also the corresponding total bitrates are given in Table 2 .

\begin{tabular}{|c|c|c|c|c|}
\hline \multirow{2}{*}{ Sequences } & \multicolumn{2}{|c|}{$\begin{array}{c}\text { Random Access } \\
\text { Configuration }\end{array}$} & \multicolumn{2}{c|}{$\begin{array}{c}\text { Low Delay B } \\
\text { Configuration }\end{array}$} \\
\cline { 2 - 5 } & $\begin{array}{c}\text { Avg.P } \\
\text { SNR }\end{array}$ & $\begin{array}{c}\text { BD- } \\
\text { Rate \% }\end{array}$ & $\begin{array}{c}\text { Avg.P } \\
\text { SNR }\end{array}$ & $\begin{array}{c}\text { BD- } \\
\text { Rate \% }\end{array}$ \\
\hline $\begin{array}{c}\text { BQSquare } \\
416 x 240\end{array}$ & 0.12 & -2.35 & 0.11 & -2.34 \\
\hline $\begin{array}{c}\text { CITY704x5 } \\
76\end{array}$ & 0.01 & -0.30 & 0.05 & -1.24 \\
\hline $\begin{array}{c}\text { PartyScene } \\
832 \times 480\end{array}$ & 0.02 & -0.98 & 0.03 & -0.77 \\
\hline $\begin{array}{c}\text { RaceHorses } \\
832 \times 480\end{array}$ & 0.01 & -0.49 & 0.05 & -1.38 \\
\hline
\end{tabular}

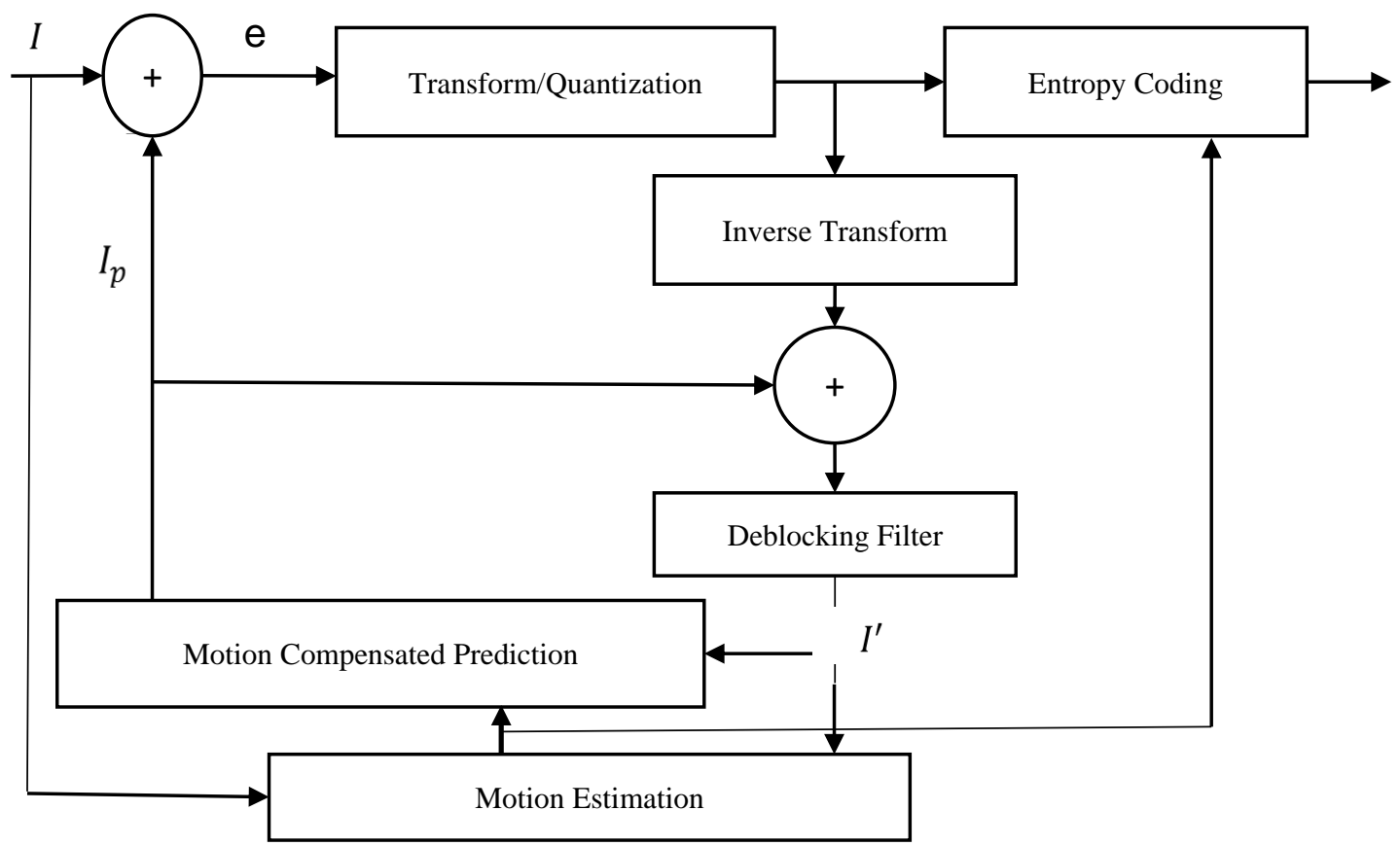

Figure (1): HEVC encoder block diagram 


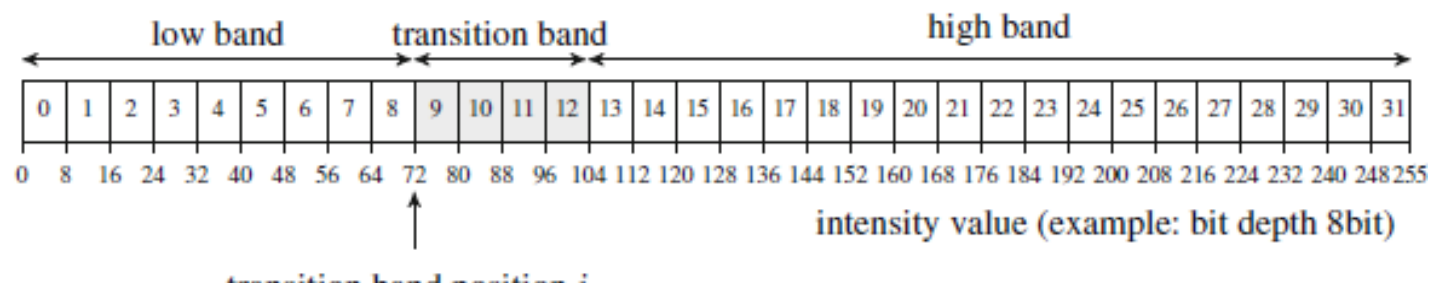

transition band position $i_{\mathrm{o}}$

Figure (2):Example for the areas of the band offset mode for SAO band position $i_{0}=9$

Table 1: BD-rate calculated using Bjontegaard rates for PSNR introduced by HEVC with SAO against HEVC without SAO for sequences.

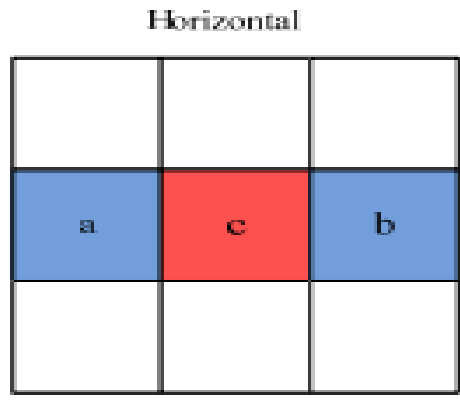

Vertical

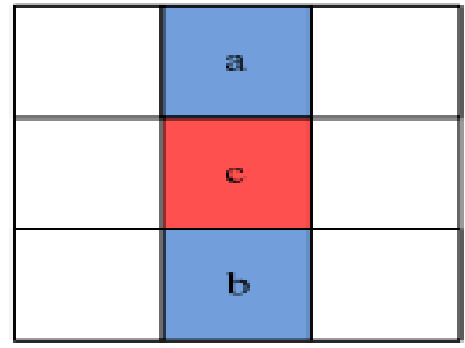

$135^{\circ}$ diagonal
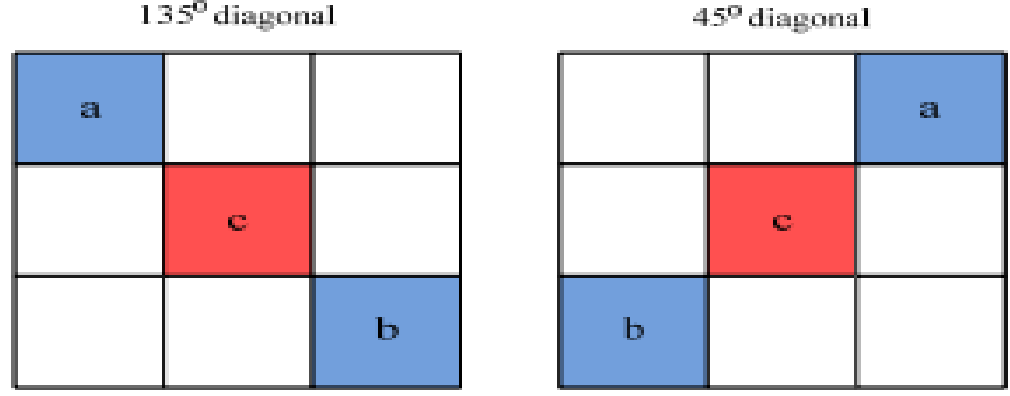

Figure (3): One dimensional three pixel patterns

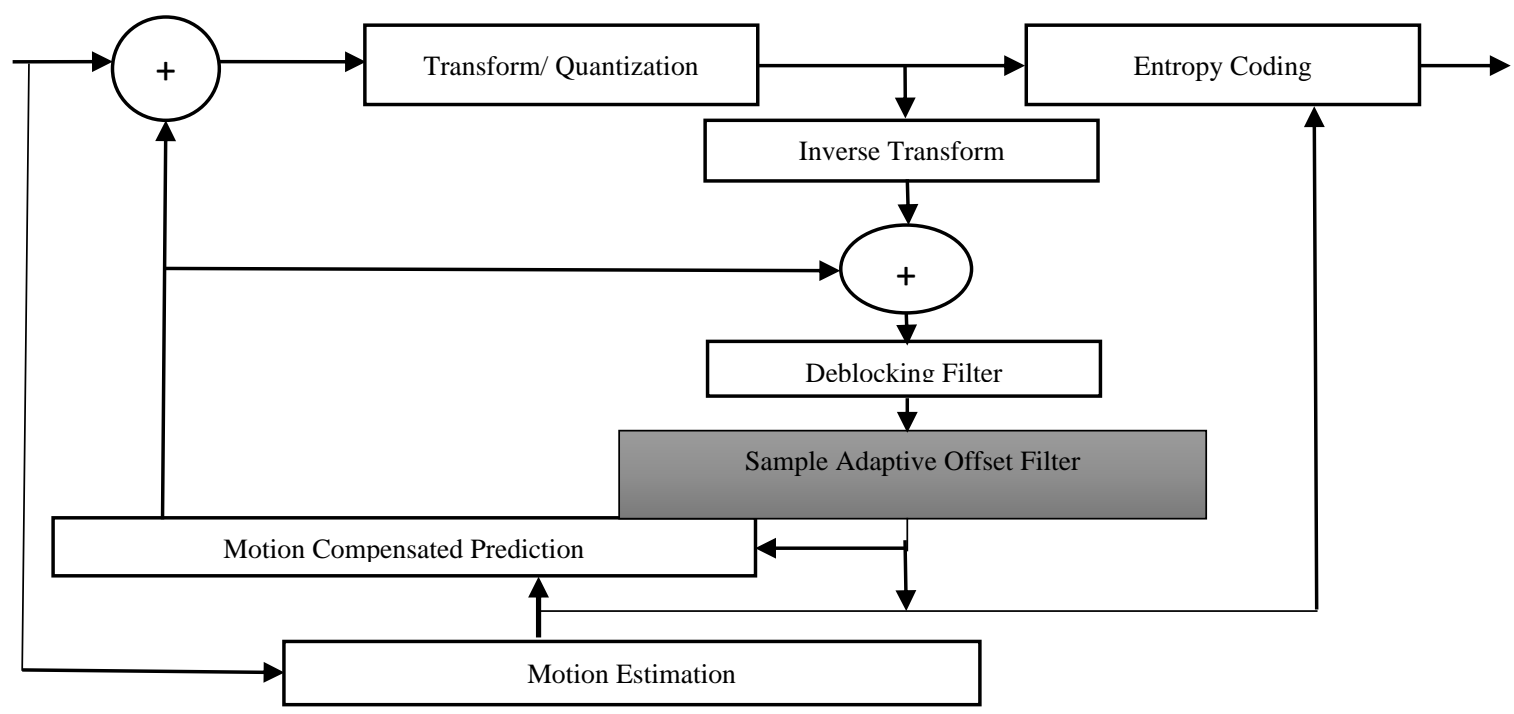

Figure 4: HEVC with SAO filter 


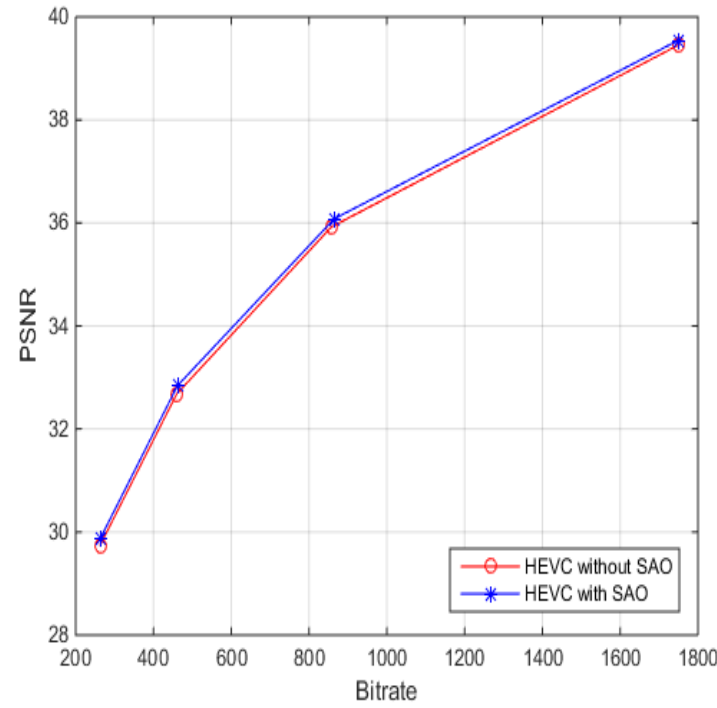

a. BQSequare video

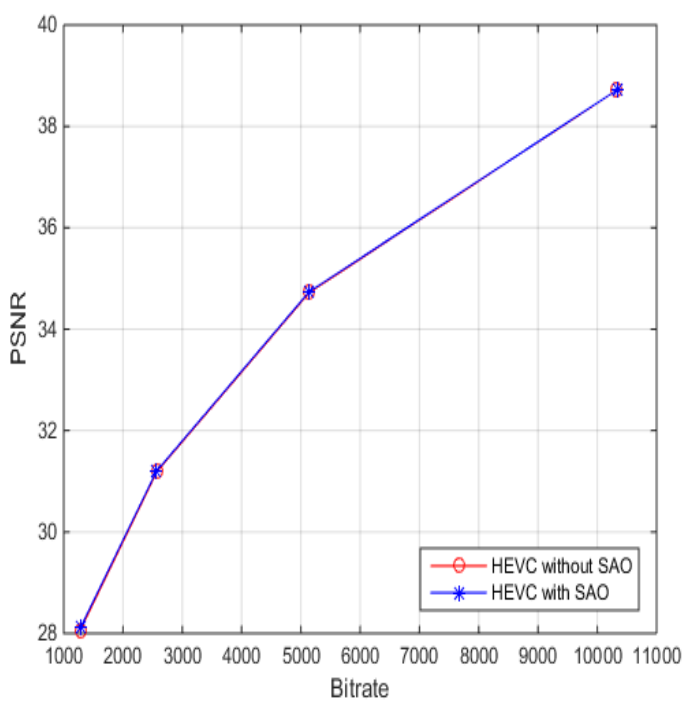

c. PartyScene video

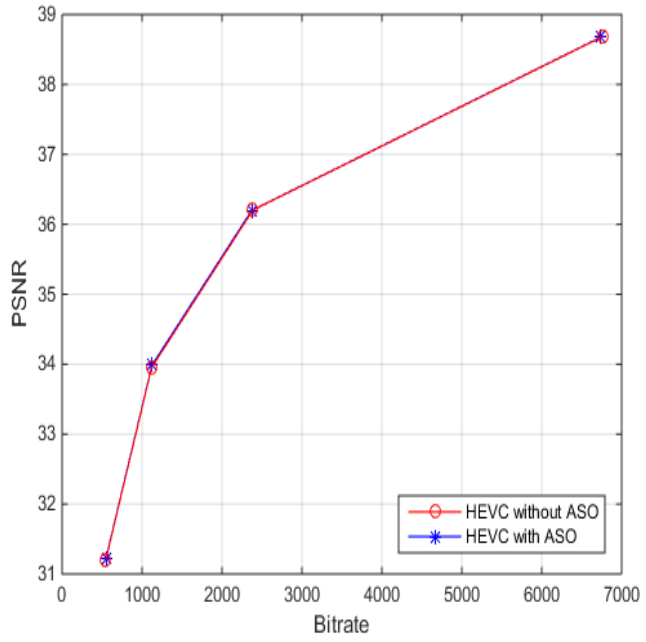

b. City video

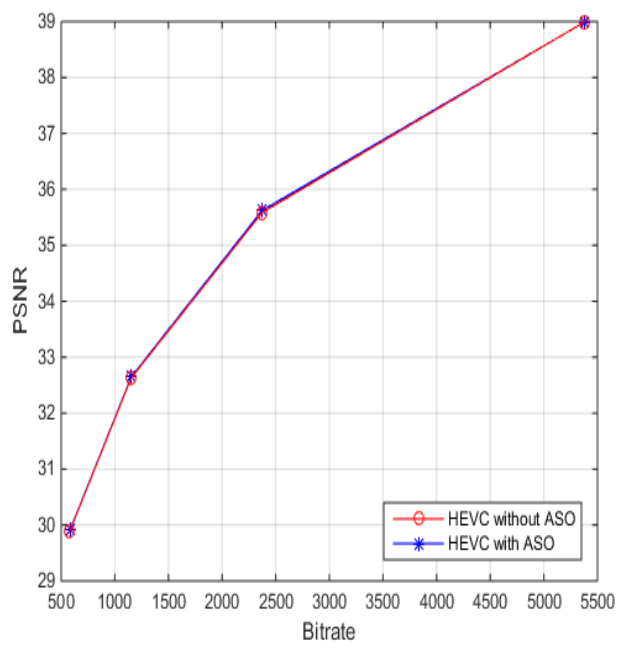

Figure (5): BD-rate of the BQSequare and PartyScene videos. 
Table 2: Experimental results for all test sequences for HEVC with SAO and HEVC without SAO.

\begin{tabular}{|c|c|c|c|c|c|c|c|c|c|c|c|}
\hline \multirow{3}{*}{ Sequence } & \multirow{3}{*}{ QP } & \multicolumn{10}{|c|}{ Low Delay Configuration } \\
\hline & & \multicolumn{5}{|c|}{ HEVC with SAO } & \multicolumn{5}{|c|}{ HEVC without SAO } \\
\hline & & trate $[\mathrm{kbit} / \mathrm{s}]$ & $\begin{array}{r}\text { YPSNR } \\
{[\mathrm{dB}]} \\
\end{array}$ & $\begin{array}{r}\text { UPSNR } \\
{[\mathrm{dB}]} \\
\end{array}$ & $\begin{array}{r}\text { VPSNR } \\
{[\mathrm{dB}]} \\
\end{array}$ & Time [sec] & $\begin{array}{l}\text { Bitrate } \\
{[\mathrm{kbit} / \mathrm{s}]}\end{array}$ & $\begin{array}{r}\text { YPSNR } \\
{[\mathrm{dB}]} \\
\end{array}$ & $\begin{array}{r}\text { UPSNR } \\
{[\mathrm{dB}]} \\
\end{array}$ & $\begin{array}{r}\text { VPSNR } \\
{[\mathrm{dB}]} \\
\end{array}$ & Time $[\mathrm{sec}]$ \\
\hline \multirow{4}{*}{ QSquare } & 22 & 2184.60 & 39.2962 & 43.4564 & 44.3278 & 294.771 & 2196.528 & 39.2403 & 43.4617 & 44.299 & 267.141 \\
\hline & 27 & 1005.864 & 35.4139 & 41.2621 & 42.0951 & 213.68 & 1011.264 & 35.3051 & 41.2353 & 42.0877 & 194.646 \\
\hline & 32 & 499.392 & 32.0702 & 39.5196 & 40.2651 & 188.02 & 500.544 & 31.9672 & 39.4987 & 40.2583 & 154.319 \\
\hline & 37 & 270.096 & 29.1056 & 38.5223 & 39.1443 & 169.733 & 268.80 & 28.973 & 38.373 & 38.9501 & 132.717 \\
\hline \multirow{4}{*}{ CITY } & 22 & 8788.248 & 39.3866 & 44.8791 & 46.9017 & 2047.474 & 8804.016 & 39.387 & 44.8171 & 46.8305 & 2339.762 \\
\hline & 27 & 2608.392 & 35.9753 & 42.9254 & 45.1949 & 1335.573 & 2612.16 & 35.9559 & 42.8747 & 45.1086 & 1578.770 \\
\hline & 32 & 1094.784 & 33.3986 & 41.3772 & 43.733 & 1033.271 & 1099.848 & 33.3688 & 41.3474 & 43.667 & 994.486 \\
\hline & 37 & 520.416 & 30.6093 & 40.3829 & 42.7496 & 884.450 & 522.96 & 30.5728 & 40.3253 & 42.6963 & 892.945 \\
\hline \multirow{4}{*}{ rtyScene } & 22 & 12172.34 & 38.8637 & 40.9853 & 41.5115 & 2147.839 & 12179.82 & 38.8697 & 40.958 & 41.4739 & 2457.554 \\
\hline & 27 & 5943.06 & 34.6996 & 38.2758 & 38.6379 & 1960.299 & 5949.28 & 34.6856 & 38.2407 & 38.609 & 1887.144 \\
\hline & 32 & 2853.14 & 30.975 & 36.4188 & 36.6133 & 1473.002 & 2857.40 & 30.9484 & 36.346 & 36.5764 & 1713.096 \\
\hline & 37 & 1357.12 & 27.7195 & 35.1978 & 35.3028 & 1245.525 & 1354.04 & 27.6754 & 35.1317 & 35.2694 & 1295.744 \\
\hline \multirow{4}{*}{ ceHorses } & 22 & 6453.276 & 39.9074 & 41.4011 & 42.3816 & 3293.036 & 6468.072 & 39.9122 & 41.3386 & 42.2665 & 7715.059 \\
\hline & 27 & 2727.96 & 35.9734 & 38.8826 & 40.0218 & 2206.91 & 2725.908 & 35.9523 & 38.79 & 39.8761 & 6047.717 \\
\hline & 32 & 1264.212 & 32.647 & 36.9813 & 38.1359 & 2921.51 & 1271.712 & 32.6175 & 36.8975 & 38.0195 & 2079.069 \\
\hline & 37 & 609.552 & 29.7733 & 35.8631 & 36.8086 & 1586.868 & 609.336 & 29.7144 & 35.7634 & 36.6691 & 1822.164 \\
\hline
\end{tabular}

\begin{tabular}{|c|c|c|c|c|c|c|c|c|c|c|c|}
\hline \multirow{3}{*}{ equence } & \multirow{3}{*}{ QP } & \multicolumn{10}{|c|}{ Random Access Configuration } \\
\hline & & \multicolumn{5}{|c|}{ HEVC with SAO } & \multicolumn{5}{|c|}{ HEVC without SAO } \\
\hline & & $\begin{array}{l}\text { Bitrate } \\
{[\mathrm{kbit} / \mathrm{s}]}\end{array}$ & PSNR [dB] & PSNR [dB] & $\begin{array}{r}\text { VPSNR } \\
{[\mathrm{dB}]}\end{array}$ & ime [sec] & $\begin{array}{l}\text { Bitrate } \\
{[\mathrm{kbit} / \mathrm{s}]}\end{array}$ & PSNR $[\mathrm{dB}]$ & $\begin{array}{r}\text { UPSNR } \\
{[\mathrm{dB}]}\end{array}$ & $\begin{array}{r}\text { VPSNR } \\
{[\mathrm{dB}]}\end{array}$ & ime [sec] \\
\hline \multirow{4}{*}{ QSquare } & 22 & 1748.736 & 39.5422 & 43.9614 & 44.8777 & 324.781 & 1751.64 & 39.4604 & 43.9482 & 44.862 & 240.974 \\
\hline & 27 & 864.936 & 36.0812 & 41.6859 & 42.4362 & 184.159 & 857.568 & 35.9231 & 41.6523 & 42.439 & 193.147 \\
\hline & 32 & 461.88 & 32.8423 & 39.8673 & 40.7357 & 164.714 & 459.84 & 32.6805 & 39.8645 & 40.6589 & 291.525 \\
\hline & 37 & 263.448 & 29.8783 & 38.6893 & 39.437 & 158.611 & 263.064 & 29.7471 & 38.602 & 39.2992 & 164.292 \\
\hline \multirow{4}{*}{ CITY } & 22 & 6735.624 & 38.6743 & 44.9846 & 47.1136 & 1893.083 & 6766.512 & 38.6874 & 44.978 & 47.1026 & 1933.68 \\
\hline & 27 & 2372.304 & 36.1988 & 43.2481 & 45.5998 & 1270.809 & 2385.744 & 36.202 & 43.229 & 45.5838 & 1281.41 \\
\hline & 32 & 1123.968 & 33.9866 & 41.5778 & 43.9669 & 855.748 & 1120.848 & 33.9533 & 41.5856 & 43.9273 & 873.434 \\
\hline & 37 & 549.0000 & 31.2278 & 40.6338 & 43.0349 & 933.557 & 544.416 & 31.1881 & 40.6042 & 43.1036 & 1036.085 \\
\hline \multirow{4}{*}{$\begin{array}{r}\text { rrtyScen } \\
\mathrm{e}\end{array}$} & 22 & 10337.00 & 38.7214 & 41.2966 & 41.8643 & 1996.895 & 10331.18 & 38.7153 & 41.2635 & 41.8626 & 2003.959 \\
\hline & 27 & 5139.44 & 34.7376 & 38.656 & 39.0673 & 1649.277 & 5144.32 & 34.7249 & 38.6312 & 39.0611 & 1715.238 \\
\hline & 32 & 2565.82 & 31.201 & 36.7817 & 37.0812 & 1535.596 & 2574.04 & 31.1938 & 36.7286 & 37.0447 & 2399.093 \\
\hline & 37 & 1279.38 & 28.1073 & 35.5283 & 35.6902 & 1272.407 & 1278.16 & 28.0592 & 35.4793 & 35.6032 & 1436.107 \\
\hline \multirow{4}{*}{ eHorses } & 22 & 5375.616 & 38.9876 & 41.3565 & 42.4438 & 2659.639 & 5381.868 & 38.9973 & 41.3358 & 42.4015 & 3830.707 \\
\hline & 27 & 2372.604 & 35.6224 & 39.2098 & 40.3742 & 2416.985 & 2371.104 & 35.5823 & 39.1494 & 40.2878 & 2248.644 \\
\hline & 32 & 1152.948 & 32.647 & 37.3874 & 38.5463 & 1893.520 & 1152.48 & 32.6288 & 37.3459 & 38.4869 & 1627.550 \\
\hline & 37 & 582.732 & 29.9149 & 36.2052 & 37.2533 & 1706.898 & 577.572 & 29.8759 & 36.1303 & 37.1918 & 1678.825 \\
\hline
\end{tabular}

\section{Conclusion}

In the paper, compare between (HEVC) with (SAO) and without a standard, that allowsimproving average PSNR and decreasing bit rate, have been presented.The performed experiments showed the average time reduction to over $1.03 \%$ in random access and $1.43 \%$ in low delay configurations for video sequences recorded when the (HEVC) with (SAO) comparing to the (HEVC) without (SAO).

\section{Acknowledgment:}

This work was supported by Al-Ma'moun University, Department of Electrical Power Techniques Engineering, which is gratefully acknowledged. 


\section{References}

[1] B. Bross, W.J. Han, G. J. Sullivan, J.R. Ohm, and T. Wiegand, "High-Efficiency Video Coding (HEVC) Text Specification Draft 9", ITU-T/ISO/IEC Joint Collaborative Team on Video Coding (JCT-VC), 2012.

[2] G. J. Sullivan, J. Ohm, W. Han, T. Wiegand, and T. Wiegand, "Overview of the high-efficiency video coding (HEVC) standard", IEEE Transactions on Circuits and Systems for Video Technology, 2012.

[3] Joint Video Team of ITU-T VCEG and ISO/IEC MPEG, ITU-T Rec. H.264, ISO/IEC 14496-10 AVC, 2005.

[4] K. Sastri, "Complexity Reduction in Inter-Layer InterPrediction in ScalableHigh Efficiency Video Coding", M.Sc. thesis, 2014.

[5] V. Sze, M. Budagavi and G. J. Sullivan, "HighEfficiency Video Coding Algorithms and Architectures," 2015.

[6] C. Fu, E. Alshina, A. Alshin, Y. Huang "Sample Adaptive Offset in the HEVC Standard", IEEE Transactions on Circuits and Systems for Video Technology, 2012.

[7] W. Shen, Y. Fan, Y. Bai, L. Huang, Q. Shang, C. Liu, and X. Zeng, "A Combined Deblocking Filter and SAO Hardware Architecture for HEVC", IEEE TRANSACTIONS ON MULTIMEDIA, 2016. 2015 .

[8] M. Wien, "High-Efficiency Video Coding", Book,

[9] M.T. Pourazad et al., "HEVC: The New Gold Standard for Video Compression", IEEE consumer electronics magazine, 2012.

[10] Joint Collaborative Team on Video Coding, "HMKTA reference software 16.6".

[11] G. Bjontegaard, "Calculation of Average PSNR Differences between RD-curves," ITU-T SG16, Doc. VCEG-M33, Austin, USA, Apr. 2001. 